\title{
Adult Classic Hodgkin Lymphoma
}

National Cancer Institute

\section{Source}

National Cancer Institute. Adult Classic Hodgkin Lymphoma. NCI Thesaurus. Code C69138.

Classic Hodgkin lymphoma that occurs in adults. 